\title{
Analysis of Mitochondrial Networks by Serial Block Face SEM
}

\author{
R.D. Leapman ${ }^{1}$, J.D. Hoyne ${ }^{1}$, B.C. Kuo ${ }^{1}$, G.N. Calco ${ }^{1}$, G. Zhang ${ }^{1}$, M.A. Aronova ${ }^{1}$ \\ ${ }^{1 .}$ National Institute of Biomedical Imaging and Bioengineering, NIH, Bethesda, MD 20892
}

Mitochondria have a central importance since they are the organelles that contain the electron transport chain and its associated molecular machinery, which synthesizes adenosine triphosphate (ATP), the key molecule that provides energy to drive chemical reactions within all eukaryotic cells. Mitochondria have other key functions too, such as heme synthesis and lipid metabolism. They are highly dynamic organelles that change their shape between discrete structures and large interconnected networks by selective fission and fusion of their membranes. The resulting fused networks can permeate through an entire cell, but their advantages, relative to isolated discrete organelles, are not fully understood. It has been suggested that networks might: (i) decrease mitochondrial degradation, (ii) dampen biochemical fluctuations, (iii) provide a mechanism for quality control and selective mitophagy, (iv) enable diffusion of proteins, and (v) transmit calcium signals and mitochondrial membrane depolarization throughout the cell [1, 2]. Although mitochondrial networks are highly dynamic, useful structural information can be obtained from electron microscopy by studying their organization at a given time point.

We have used a Sigma VP (Zeiss Inc.) scanning electron microscope (SEM), equipped with a 3View (Gatan Inc.) serial block face (SBF) system [3], to quantify the connectivity of mitochondrial networks in entire insulin-secreting $\beta$-cells of mouse pancreatic islets of Langerhans. Previous work has suggested that alterations in mitochondrial fission and fusion might play a role in nutrientinduced $\beta$-cell apoptosis with possible involvement in the pathophysiology of Type 2 diabetes [4].

In our previous SBF-SEM studies [5,6], manual segmentation of mitochondria in a single $\beta$-cell took about one week for a single operator, which precludes analysis of multiple cells within a reasonable time. We have explored a faster and more practicable approach for segmenting mitochondria by making use of tools within the Amira visualization software package (FEI Inc.) to partially automate the segmentation process, so that an entire cell could be analyzed in approximately two hours. This procedure included: $4 \times 4$ binning of each block face image, thresholding the intensity within the more heavily stained mitochondrial regions (Fig. 1A), and erasing manually remaining islands of intensity. Results of the semi-automated segmentation (Fig. 1B) were compared with fully manual segmentation, which showed that the total mitochondrial volumes differed by $2 \%$, whereas the number of networks differed by $18 \%$ ( 76 for manual, and 63 for semi-automated segmentation in the analyzed cell). Using this approach we were able to analyze quantitatively mitochondrial networks in individual pancreatic $\beta$-cells in terms of the total mitochondrial volume, average volume per network, total network length, and average network length (Fig. 2A). Analysis of $7 \beta$-cells gave the distribution of network volumes as a function of network length in Fig. 2B. This revealed that one-third of the mitochondrial volume was contained in networks of length $<2.6 \mu \mathrm{m}$, one-third in networks of length between $2.6 \mu \mathrm{m}$ and $10.4 \mu \mathrm{m}$, and one-third in highly fused networks of length between $10.4 \mu \mathrm{m}$ and $60 \mu \mathrm{m}$.

In conclusion, SBF-SEM provides quantitative data on the organization of mitochondrial networks, which makes it possible to test computational models of mitochondrial fusion and fission [7]. 
References:

[1] H. Hoitzing et al, Bioessays 37 (2015) p. 687.

[2] B. Clancy et al, Nature 523 (2015) p. 617.

[3] W. Denk and H. Horstmann, PLoS Biol. 2 (2004) p. 1900.

[4] A.J.A. Molina et al, Diabetes 58 (2009) p. 2303.

[5] C.R. Pfeifer et al, J. Struct. Biol. 189 (2015) p. 44.

[6] A. Shomorony et al, J. Microsc. 259 (2015) p. 155.

[7] The authors thank Drs. A.L. Notkins, T. Cai, and H. Xu for providing the specimens of pancreatic islets of Langerhans. This research was supported by the intramural program of the National Institute of Biomedical Imaging and Bioengineering, NIH.

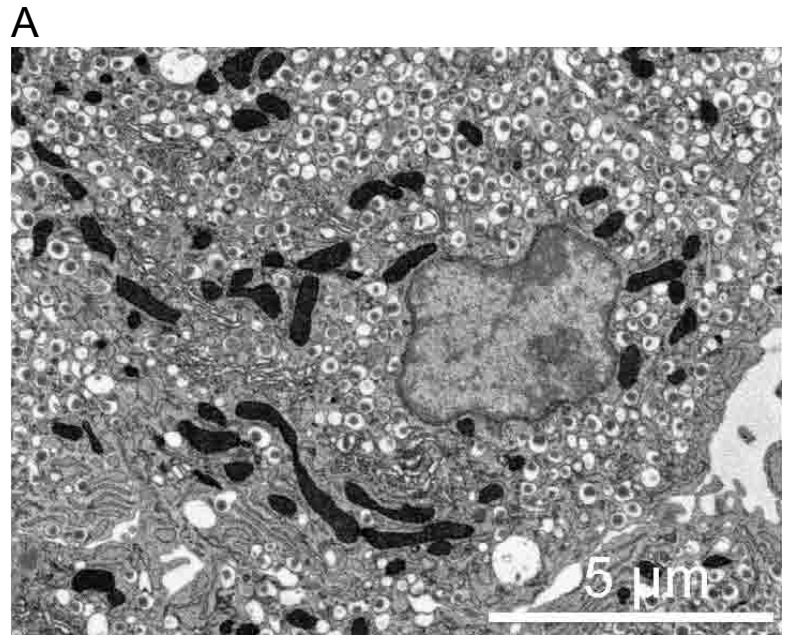

B

Figure 1. (A) SBF-SEM image from region of wild-type mouse pancreatic islet $\beta$-cell showing cross sections through heavily stained mitochondria. (B) Semi-manual segmentation of 110 mitochondrial networks in single cell; each network is indicated in a separate color. Although cell appears crowded with networks, the mitochondria occupy less than $10 \%$ of the total cell volume.

A

\begin{tabular}{|l|c|}
\hline \multicolumn{2}{|c|}{$\begin{array}{c}\text { Analysis of mitochondrial network per } \\
\beta \text { cell of mouse pancreatic islet }\end{array}$} \\
\hline $\begin{array}{l}\text { Total mitochondrial } \\
\text { volume per cell }\left(\mu \mathrm{m}^{3}\right)\end{array}$ & $93 \pm 19$ \\
$\begin{array}{l}\text { Average mitochondrial } \\
\text { volume per network }\left(\mu \mathrm{m}^{3}\right)\end{array}$ & $0.8 \pm 0.1$ \\
$\begin{array}{l}\text { Total mitochondrial } \\
\text { network length per cell }(\mu \mathrm{m})\end{array}$ & $258 \pm 52$ \\
$\begin{array}{l}\text { Average mitochondrial } \\
\text { network length }(\mu \mathrm{m})\end{array}$ & $2.3 \pm 1.1$ \\
\hline
\end{tabular}

$\mathrm{B}$

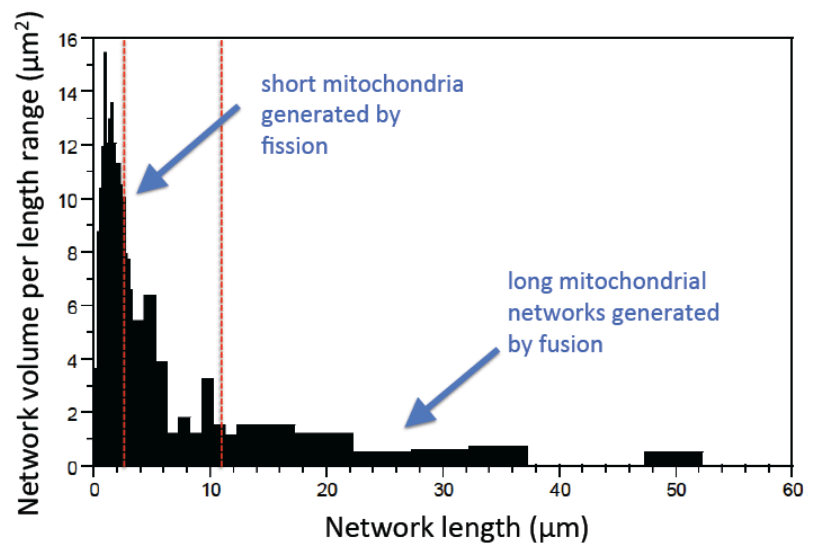

Figure 2. (A) Quantitative analysis of mitochondrial networks in 7 pancreatic $\beta$-cells showing, total mitochondrial volume, average volume per network, total network length, and average network length. (B) Distribution of mitochondrial network volumes as a function of network length for a total of 7 pancreatic $\beta$-cells; the vertical red lines indicate the approximate network lengths containing one-third and two-thirds of mitochondrial volume. 